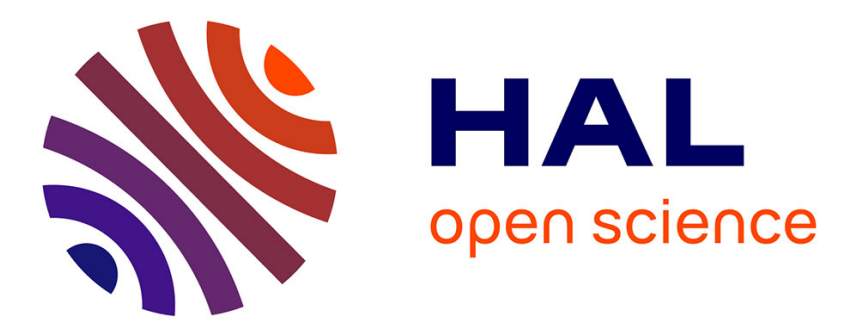

\title{
Laser-driven proton scaling laws and new paths towards energy increase
}

J. Fuchs, P. Antici, E. d'Humières, E. Lefebvre, M. Borghesi, E. Brambrink, C. A. Cecchetti, M. Kaluza, Victor Malka, M. Manclossi, et al.

\section{- To cite this version:}

J. Fuchs, P. Antici, E. d'Humières, E. Lefebvre, M. Borghesi, et al.. Laser-driven proton scaling laws and new paths towards energy increase. Nature Physics, 2006, 2 (1), pp.48-54. 10.1038/nphys199. hal-00502703

\section{HAL Id: hal-00502703 \\ https://hal-polytechnique.archives-ouvertes.fr/hal-00502703}

Submitted on 17 Aug 2021

HAL is a multi-disciplinary open access archive for the deposit and dissemination of scientific research documents, whether they are published or not. The documents may come from teaching and research institutions in France or abroad, or from public or private research centers.
L'archive ouverte pluridisciplinaire HAL, est destinée au dépôt et à la diffusion de documents scientifiques de niveau recherche, publiés ou non, émanant des établissements d'enseignement et de recherche français ou étrangers, des laboratoires publics ou privés. 


\section{Laser-driven protons scaling laws and new paths towards energy increase}

J. Fuchs ${ }^{1,2, *}$, P. Antici ${ }^{1,2,11,12}$, E. d'Humières ${ }^{3}$, E. Lefebvre ${ }^{3}$, M. Borghesi ${ }^{4}$, E. Brambrink ${ }^{1}$, C.A. Cecchetti $^{4}$, M. Kaluza ${ }^{5}$, V. Malka ${ }^{6}$, M. Manclossi ${ }^{6,7}$, S. Meyroneinc ${ }^{8}$, P. Mora $^{9}$, J. Schreiber ${ }^{5}$, T. Toncian $^{10}$, H. Pépin ${ }^{11}$, P. Audebert ${ }^{1}$

${ }^{1}$ Laboratoire pour l'Utilisation des Lasers Intenses, UMR 7605 CNRS-CEA-École PolytechniqueUniversité Paris VI, Palaiseau, France

${ }^{2}$ Physics Department, MS-220, University of Nevada, Reno, Nevada 89557, USA

${ }^{3}$ DPTA, CEA-DIF, Bruyères-le-Châtel, France

${ }^{4}$ School of Mathematics and Physics, The Queen's University, Belfast, United Kingdom

${ }^{5}$ Max-Planck-Institut für Quantenoptik, 85748 Garching, Germany

${ }^{6}$ Laboratoire d'Optique Appliquée - ENSTA, UMR 7639, CNRS, Ecole Polytechnique, 91761 Palaiseau,

France

${ }^{7}$ Dipartimento di Fisica "G.Occhialini" and INFM, Università di Milano-Bicocca, Italy

${ }^{8}$ Centre de Protonthérapie d'Orsay, BP 65, 91402 Orsay, France

${ }^{9}$ Centre de Physique Théorique, UMR 7644 CNRS-Ecole Polytechnique, 91128 Palaiseau, France

${ }^{10}$ Institut für Laser und Plasmaphysik, Heinrich-Heine-Universität, Düsseldorf, Germany

${ }^{11}$ INRS-ÉMT, 1650 bd. L. Boulet, J3X1S2 Varennes, Québec, Canada

${ }^{12}$ Dipartimento di Energetica, Università di Roma «La Sapienza », Via Scarpa 14-16, 00161 Roma, Italy

* corresponding author: julien.fuchs@polytechnique.fr 
Recent years have seen remarkable progress in the development of laser-based particle accelerators. The ability to produce ultra-bright beams of multi-MeV protons routinely has many potential uses from engineering to medicine, but for this potential to be realized substantial improvements in the performances of these devices must be made. Here we show that in a laserdriven accelerator regime that has been demonstrated experimentally to produce the highest energy protons, scaling laws derived from fluid models and supported by numerical simulations can be used to accurately describe the acceleration of proton beams for a large range of laser and target parameters. This enables us to evaluate the laser parameters needed to produce high-energy and high quality proton beams of interest for radiography of dense objects or proton therapy of deep-seated tumours.

Energetic proton beams with high beam quality have been produced in the last five years from thin metallic foils (usually aluminium) irradiated by ultra intense short laser pulses [1,2,3]. Protons accelerated from solids originate primarily from contaminant layers of water vapour and hydrocarbons on the target surface [4].

These proton beams are extremely laminar $[5,6]$, quite collimated $\left(\sim 15^{\circ}\right.$ half-angle with a divergence decreasing with the beam energy) with a smooth angular distribution [7] and have a duration at the source of the order of a picosecond. Due to these qualities, these beams are already being considered or applied in high-resolution charged-particle radiography [8], or production of high energy density matter of interest for astrophysics [9], and could also lead to high-brightness injectors for accelerators [5] or sources for protontherapy [10,11,12] or radioisotope production [13].

However, these present-day sources are not yet optimized for the intended applications. The determination of the scaling laws discussed here is a necessary step to achieve this optimization. Several scaling studies have already been carried out on different facilities (from small table top lasers to single pulse large laser facilities) $[14,15,16,17,18,19,20,21]$. However, since they cannot be 
fully compared due to different sets of parameters used, no clear picture has yet emerged of the relative importance of the various target (thickness) and laser parameters (pulse energy, pulse duration, peak intensity, and focal spot size).

Here we present the results of a series of experiments on aluminium foils, measuring the proton beam maximum energy and energy conversion efficiency as a function of varying laser and target parameters. The experimental scaling laws compare favourably with the prediction of a simple fluidbased model of proton acceleration. Two dimensional Particle-in-Cell (PIC) simulations illuminate and support the successful use of the fluid model. The fluid model emerges as a helpful predictive tool for high energy and high quality proton beam production of interest, e.g. for radiography of dense objects or for protontherapy.

Finally, we discuss alternative paths for the central task of increasing the maximum energy of the proton beam, with particular attention to a new regime using volumetric heating of ultra-thin targets by ultra-high temporal contrast laser pulses.

\section{Acceleration mechanisms}

Up to the presently achievable maximum laser intensities, the basic mechanism involved in the production of these proton beams is electrostatic acceleration of protons at the target rear (non irradiated) surface $[22,23,24]$. At higher intensities, numerical simulations (discussed below) show that this mechanism is still effective. The proton acceleration is achieved by charge separation electric fields induced by the laser-accelerated electrons produced at the front surface going through the target and emerging from the rear.

For completeness we mention two other ways in which fast protons may be produced at sufficiently high intensities, but whose scaling will not be discussed, failing any experimental testing 
of the concepts. First, at higher laser intensities, i.e. above $\sim 10^{21} \mathrm{~W} . \mathrm{cm}^{-2}$, numerical simulations seem to indicate that laser-induced ion shocks could also accelerate protons to high energies [25]. However, simulations also show that fewer protons are produced than by acceleration at the target rear-surface, with lower beam quality and with large fluctuations in the maximum beam energy due to the instabilities to which this mechanism is linked [26]. Secondly, also according to simulations, for extreme intensities approaching $10^{23} \mathrm{~W} \cdot \mathrm{cm}^{-2}$, the interaction exhibits a continuous transition from the rear-surface acceleration/shock acceleration regimes to a regime where the radiation pressure of the electromagnetic wave is directly converted into ion energy via the space-charge force related to the displacement of the electrons in a thin foil [27]. In this regime the proton energies could reach GeVs and the efficiency of the laser energy conversion into fast ion energy could be much higher than projected from what is presently known. This transition remains however to be observed experimentally.

Since applications require robustness and high beam quality, we will in this work concentrate on the rear-surface mechanism that has been experimentally proven in several facilities and that has shown to satisfy these two criteria.

\section{Experimental results and modelling}

In the series of experiments reported here, we have measured the proton spectra accelerated from laser-irradiated solid aluminium targets while varying only one parameter at a time, either laser intensity $(I)$, or laser energy $(E)$, or laser pulse duration $(\tau)$ or target thickness (d). Other conductor targets (e.g. $\mathrm{Au}$ ) give similar proton beam results to those using Al. Insulator targets on the other hand display unsatisfactory filamentary proton beams [7]. Hence aluminium targets are all that are studied here. We 
have compared simultaneously all the obtained scalings with a simple self-similar, isothermal, timelimited fluid model using a single free parameter, the effective acceleration time (or limit time) $t_{\text {acc }}$. As we will see below, $\mathrm{t}_{\mathrm{acc}} \sim 1.3 \tau$ matches well with all the scalings results we obtained.

The energy spectrum presently observed both in experiments and in simulations can be approximated by a quasi-thermal distribution with a sharp cut-off at a maximum energy. Typical spectra observed in the experimental conditions reported here can be seen in Ref. [24,21].

The maximum (cut-off) energy that can be gained by the accelerated ions based on the simple selfsimilar, isothermal, fluid model, e.g. Eq. 10 of Ref. [28]) is given by:

$$
\mathrm{E}_{\max }=2 * \mathrm{~T}_{\mathrm{hot}} *\left[\ln \left(\mathrm{t}_{\mathrm{p}}+\left(\mathrm{t}_{\mathrm{p}}{ }^{2}+1\right)^{1 / 2}\right)\right]^{2}
$$

where $t_{\mathrm{p}}=\omega_{\mathrm{pi}} * \mathrm{t}_{\mathrm{acc}} /(2 * \exp [1])^{1 / 2}$ is the normalized acceleration time, normalized using the ion (of charge number $Z_{\mathrm{i}}$ and mass $\mathrm{m}_{\mathrm{i}}$, here protons with $Z_{\mathrm{i}}=1, m_{\mathrm{i}}=m_{\mathrm{p}}$ ) plasma frequency $\left.\omega_{\mathrm{pi}}=\left[\left(\mathrm{Z}_{\mathrm{i}} * \mathrm{e}^{2 *} \mathrm{n}_{\mathrm{e} 0}\right)\right) /\left(\mathrm{m}_{\mathrm{i}} * \varepsilon_{0}\right)\right]^{1 / 2}$ with $\mathrm{t}_{\mathrm{acc}}$ the effective acceleration time, and $\mathrm{T}_{\text {hot }}$ and $\mathrm{n}_{\mathrm{e} 0}$ the temperature and density of the hot electrons that drive the rear-surface expansion. This model updates previous models of freely expanding plasma [29] with a steady electron temperature (and thus unlimited acceleration) to the case of a sudden burst of energetic electrons. As our simple model cannot take into account the progressive transfer of energy from the fast electrons to the ions and the decrease of the accelerating charge separation field, we use the crude approximation of simply fixing the acceleration time in a way dictated by the laser pulse length. We will see below that, consulting the results of the PIC simulations, although not physically accurate, this approximation works well.

As in most of the experiments, a pre-formed plasma (pre-plasma) was present in front of the target due to long-duration, low-level laser energy reaching the target before the main pulse. Following the irradiation by the main pulse, the temperature of the fast electrons produced in this preplasma $T_{\text {hot }}$ is found numerically and experimentally to be given by the laser ponderomotive potential $T_{p}$ 
$=\mathrm{m}_{\mathrm{e}} \mathrm{c}^{2}\left(\left[1+\mathrm{I} \lambda_{\mathrm{sm}}{ }^{2} / 1.37 \times 10^{18} \mathrm{Wcm}^{-2} \mu \mathrm{m}^{2}\right)^{1 / 2}-1\right]$ where $\mathrm{m}_{\mathrm{e}}$ is the electron mass, $\mathrm{I}$ is the laser power density ("intensity") in W.cm ${ }^{-2}$ and $\lambda_{\mathrm{m}}$ is the laser wavelength in microns [30,31]. We estimate $\mathrm{n}_{\mathrm{e} 0}$ by considering that the electrons accelerated at the target front surface are ballistically sprayed into the target. The total number of electrons accelerated into the target is $\mathrm{N}_{\mathrm{e}}=\mathrm{fE} / \mathrm{T}_{\mathrm{p}}$ where $\mathrm{f}$ is the fraction of laser light that is absorbed into the preplasma as fast electrons. This fraction $\mathrm{f}$ has been found to depend on the laser incident intensity as follows: $\mathrm{f}=1.210^{-15} \mathrm{I}^{0.74}\left[\mathrm{~W} / \mathrm{cm}^{2}\right]$ with a maximum of 0.5 [32,33]. Since the $\mathrm{N}_{\mathrm{e}}$ electrons are accelerated over the laser pulse duration and spread over the surface of the sheath $S_{\text {sheath }}$, we have $n_{e 0}=N_{e} /\left(c \tau S_{\text {sheath }}\right)$ with $S_{\text {sheath }}=\pi\left(r_{0}+d \times \tan \theta\right)^{2}$. The latter depends on the half-angle divergence $\left(\theta \sim 25^{\circ}\right)$ of the hot electron inside the target [7], the target thickness $\mathrm{d}$, and the initial radius $r_{0}$ of the zone over which the electron are accelerated at the target front-surface, i.e. the laser spot. Note that the values of the sheath extension obtained with this model (e.g. $\sim 25 \mu \mathrm{m}$ for a $18 \mu \mathrm{m}$ thick target) are in good agreement with direct measurements of the sheath size (see Figure 2.c of Ref. [5]). Finally, an effective "acceleration time", $\mathrm{t}_{\mathrm{acc}}$, has to be defined. Otherwise, because of its isothermal hypothesis (i.e. no energy depletion of the electrons), the fluid model of Ref. [28] would predict endless proton acceleration.

The fluid model also gives the number of accelerated protons per unit energy: $d N / d \mathrm{E}=n_{e 0} c_{s} t_{a c c} \mathrm{~S}_{\text {sheath }} /\left(2 \mathrm{ET} T_{\text {hot }}\right)^{1 / 2 *} \exp \left(-\left(2 \mathrm{E} / T_{\text {hot }}\right)^{1 / 2}\right)$

We can thus compare the experimental value of the laser to proton energy conversion efficiency to the one given by the model. Both are obtained by integrating the spectrum between a fixed minimal energy, here chosen to be $4 \mathrm{MeV}$, and the maximum cut-off energy, and by dividing this number by the laser initial energy. 

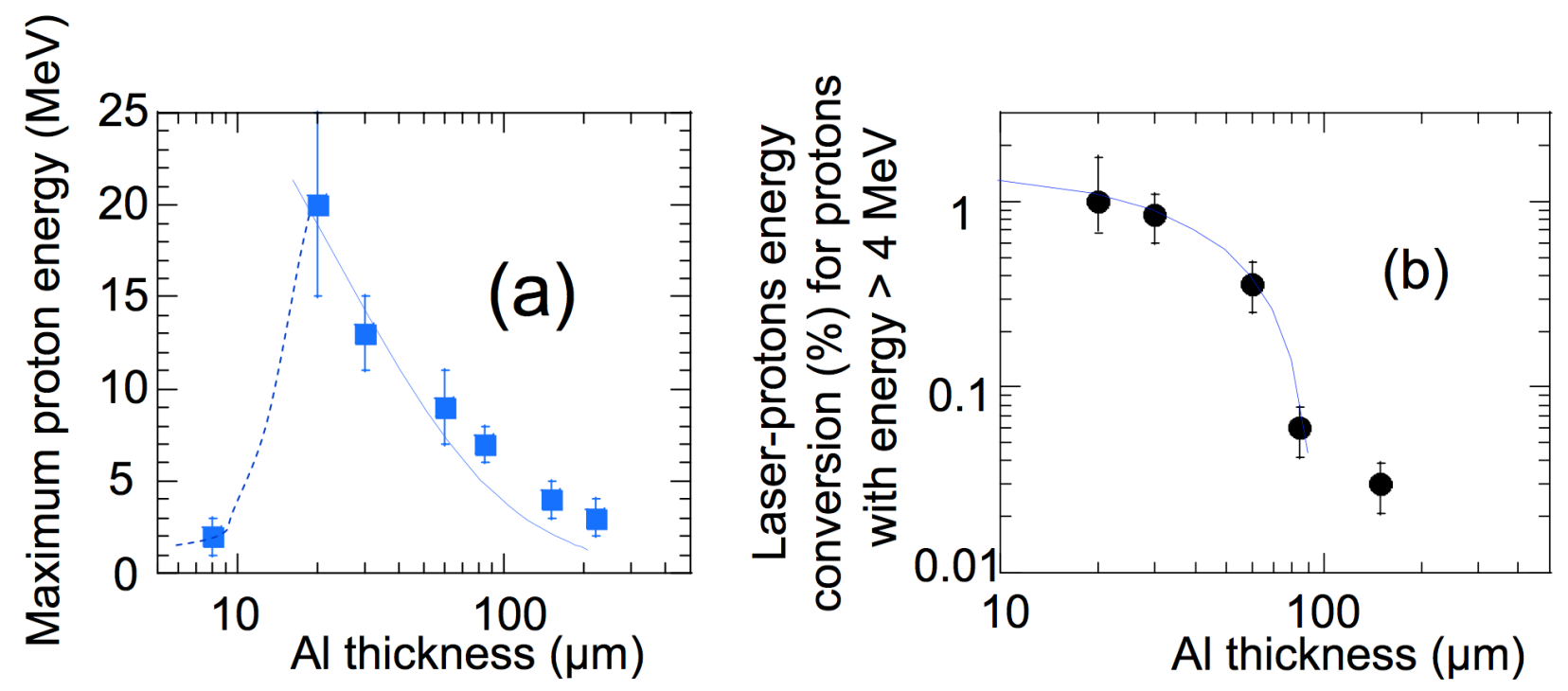

Figure 1, a decrease in target thickness results in an increase in the maximum proton energy and in the energy conversion efficiency. However, if the target is too thin $(\leq 8 \mu \mathrm{m}$ for the parameters of
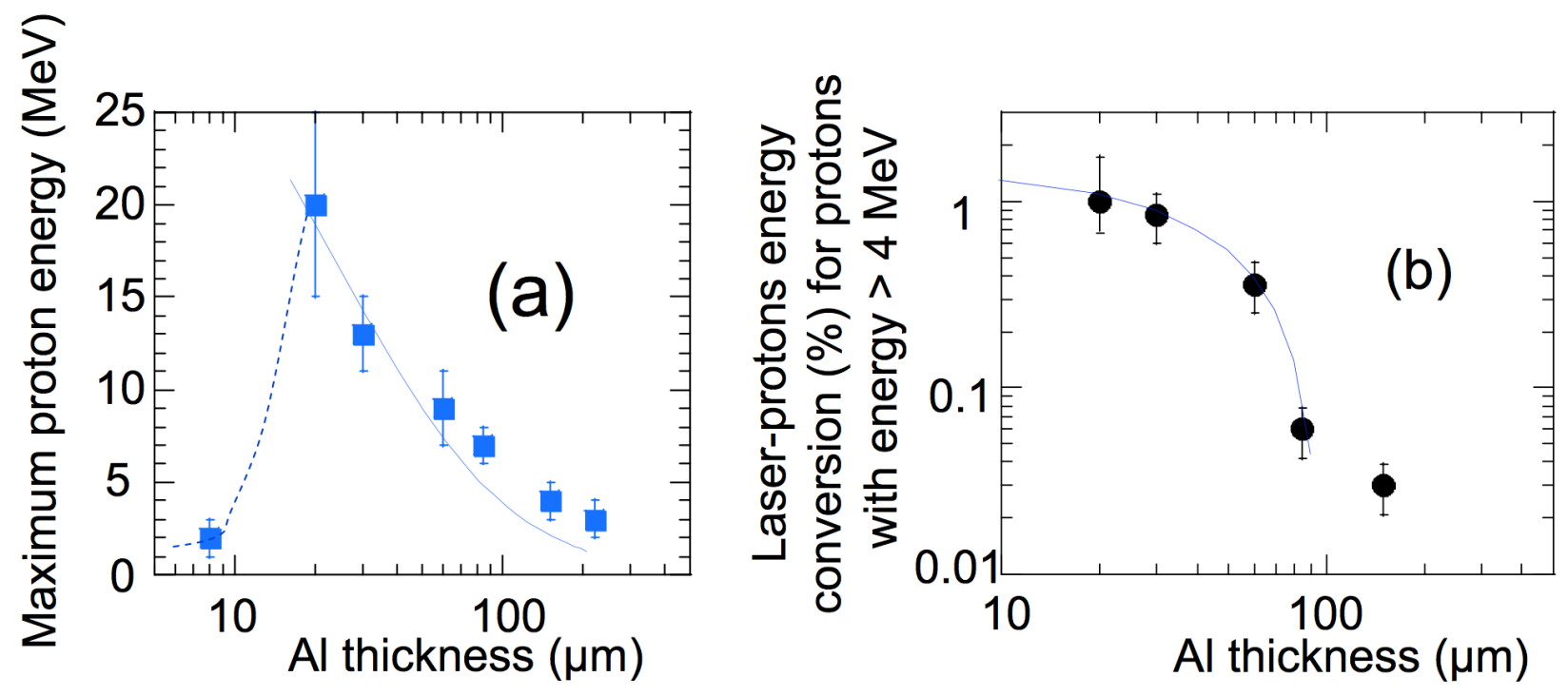

Figure 1), protons are not accelerated to high energies. This is consistent with the fact that for targets which are too thin, the rear-surface is massively perturbed by the shock wave launched $\sim 1$ ns before the main pulse by the laser amplified spontaneous emission (ASE) [21]. For the thicker targets of 
interest here, this slow shock wave preheating does not have the time to reach the rear surface before the hot electron sheath formation. Using Eq. (1) to compute $\mathrm{n}_{\mathrm{e} 0}$ and Eq.(2) to compute the laser-toproton conversion efficiency, we find a good agreement with the experimental data taking, as $\begin{array}{llllll}\text { mentioned } & \text { above, } & \mathrm{t}_{\mathrm{acc}}=1.3 \times \tau & \text { This } & \text { is } & \text { shown }\end{array}$
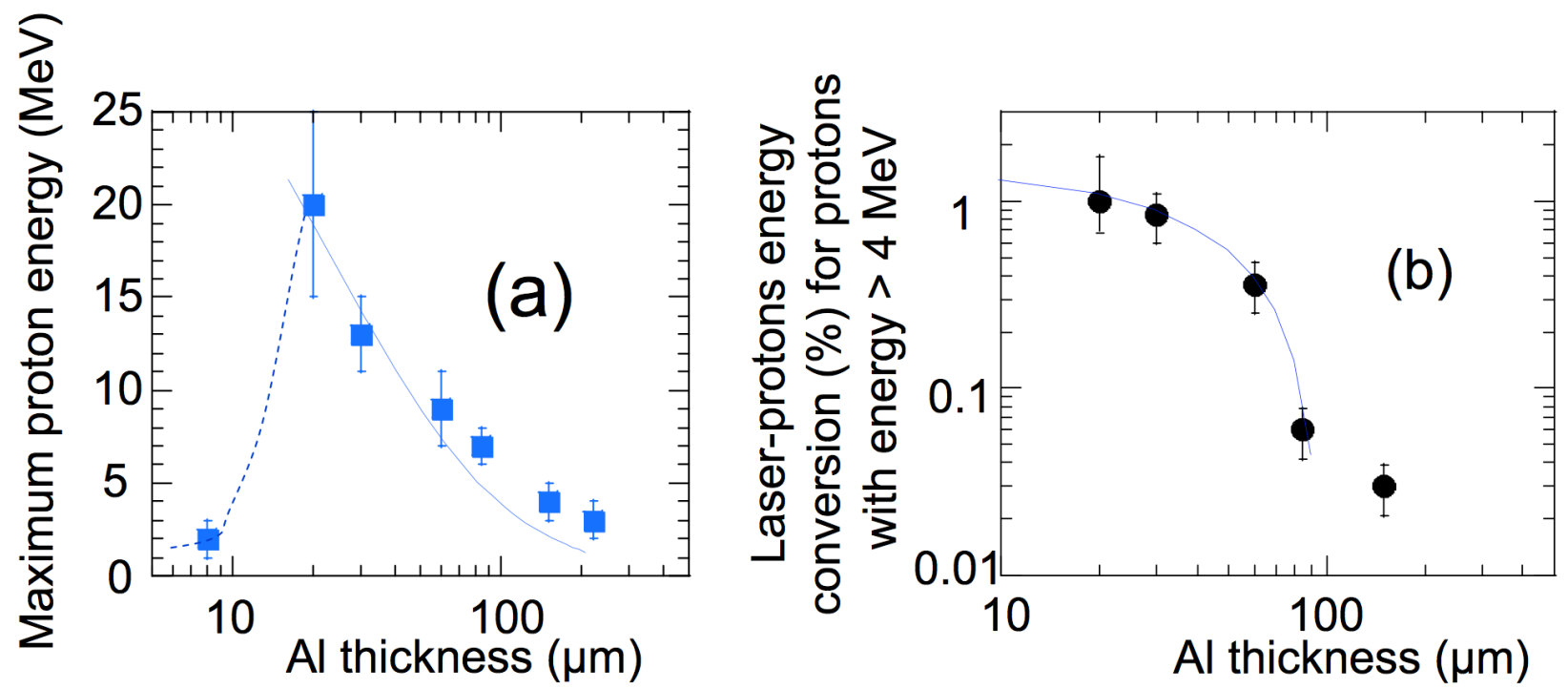

Figure 1.

Good agreement is also found between the isothermal fluid model and the measurements when studying the dependence of the proton beam energy characteristics with laser energy (keeping the pulse duration constant) as shown in Figure 2, or laser pulse duration (keeping the laser intensity constant) as shown in Figure 3. For both of these, the targets are 25 microns thick Al foils.

Figure 2.b shows that the conversion efficiency increases more with laser energy than the laser energy does when the pulse duration is kept constant. This is due to the fact that when one increases the laser energy, the proton spectrum extends to higher energies and that the number of particles also scales up. This means, for instance, that one would obtain more energy in the proton beam with a single shot at $10 \mathrm{~J}$ rather than cumulating 10 shots at $1 \mathrm{~J}$. 
Regarding Figure 3.b, we note that the model gives, in the case of long laser pulses, lower conversion efficiency compared to the measured ones. As the maximum proton energy is well modelled, this is due to the spectral slope that is underestimated by the model, maybe due to recirculation effects [20] that may become important at long pulse durations.

We also find the same good agreement between experimental data and the model when we vary the pulse duration while keeping the laser energy constant; here the dependence of the maximum proton energy is rather weak with the laser pulse duration.

Note that in a complementary regime of shorter pulses, 55 to $400 \mathrm{fs}$, Oishi et al. [19] have explored the dependence of the proton maximum energy with laser energy and pulse duration. It is interesting to note that their experimental trends agree with the fluid model.

The model (still using $t_{a c c}=1.3 \times \tau$ ) also fits well with previously published measurements performed at various laser facilities as shown in Figure 4. Considering the variety of experimental configurations, we have selected only data in similar parameter ranges in the two plots of Figure 4. Figure 4.a shows the maximum proton energy evolution versus the laser pulse duration, for two intensity ranges (circles and squares) together with the prediction of the fluid model for various intensities (lines). Comparing the laser to proton energy conversion efficiency between the same experiments and the model is more difficult since published works do not use the same proton energy range over which the spectrum is integrated. For this reason, we have displayed in Figure 4.b the proton number for a $1 \mathrm{MeV}$ bin centred at $10 \mathrm{MeV}$, for two laser pulse durations (circles and squares) together with the prediction of the fluid model using a pulse duration of $0.5 \mathrm{ps}$.

Finally, in order to validate further the use of the fluid model and to obtain more details on the acceleration mechanisms, we have performed two-dimensional PIC simulations of rear-side proton acceleration with parameters similar to the experimental ones. As expected, these kinetic simulations 
show, as illustrated in Figure 5.a, that acceleration is far from being isothermal, and is slower than in the fluid model. Nonetheless, Figure 5.b shows that the maximum proton energy in the PIC simulation saturates at a value equal to the isothermal model prediction for a time of the order of the incident pulse duration. This numerical observation supports and explains the successful use of the isothermal model in fitting the experimental energy values. In addition, we have compared the fluid model and the PIC simulations for higher laser intensities than used in the experiments. The comparison is favourable, as can be seen in Table 1.

\begin{tabular}{|l|l|l|l|l|l|}
\hline I & Laser & Focal & Target & Proton & Proton \\
$\left.{ }^{2}\right)$ & pulse & spot & thickness & $\mathrm{E}_{\max }$ & $\mathrm{E}_{\max }$ \\
duration & FWHM & $(\mu \mathrm{m})$ & from & from \\
PIC $)$ & $(\mu \mathrm{m})$ & & $\begin{array}{l}\text { model } \\
(\mathrm{MeV})\end{array}$ & $(\mathrm{MeV})$ \\
\hline $10^{22}$ & 36 & 6 & 2 & 319 & 368 \\
\hline $210^{21}$ & 36 & 6 & 2 & 111 & 108 \\
\hline $10^{21}$ & 36 & 6 & 2 & 60 & 63 \\
\hline $310^{20}$ & 36 & 6 & 1 & 37 & 27 \\
\hline $310^{20}$ & 150 & 6 & 4 & 72 & 82 \\
\hline $310^{19}$ & 320 & 6 & 19 & 16 & 13 \\
\hline
\end{tabular}

Table 1: Comparison between maximum proton energy observed in 2D PIC simulations and calculated by the fluid model. The comparison is made mainly at 36 fs laser pulse duration for practical computational time limitations. 
3. Application for proton energy increase

Several of the applications require a significant proton energy increase compared to what has been achieved up to now. A first example is the radiography of dense objects either static or dynamic. In the particular case of interest for inertial confinement fusion, a laminar flux of protons of $60 \mathrm{MeV}$ or more would be appropriate to probe compressed pellets of $100 \mu \mathrm{m}$ diameter. Regarding protontherapy, the production of energetic proton beams of high beam quality with lasers could provide a promising alternative to the present use of conventional accelerators. The interest of using lasers would be to take advantage of a potential significant reduction of size and cost of treatment facilities that would allow spreading them in the hospital environment. In order to pursue such an application, the proton energy has to be high enough to penetrate through several $\mathrm{cm}$ of tissue, i.e. 60 $\mathrm{MeV}$ corresponding to eye tumours or to tumours in small animals for preclinical studies, and 200 $\mathrm{MeV}$ corresponding to the deepest zones to be treated $(25 \mathrm{~cm})$.

Using the fluid model as a predictive tool, we can calculate the laser parameters that would be needed to achieve, in particular, a proton beam with a maximum energy of $200 \mathrm{MeV}$. This is shown in Figure 6 for various target thicknesses, laser pulse durations, and laser spot sizes. According to Figure 6.a, there seems to be an optimum for the pulse duration in the range $\sim 200 \mathrm{fs}-1$ ps in order to take full advantage of the proton acceleration time. This is quite longer than what has been previously considered in simulation feasibility studies for protontherapy since these studies have restricted themselves to much shorter pulses $[10,11]$.

To achieve practical protontherapy treatment, there are two further requirements: (i) the delivered dose requirement and (ii) a sufficiently small energy spread $\Delta \mathrm{E} / \mathrm{E}<<1$ of the beam. Of course, as we 
have mentioned there is a continuous spectrum of fast protons below the maximum energy, with flux increasing rapidly as the energy decreases. However several solutions for energy selection from this continuum have already been suggested $[34,35]$, so we chose not to repeat that material here. Regarding the issue of the available proton flux per laser shot, we can use the scaling of the proton number with the laser intensity of Figure $4 . \mathrm{b}$ to deduce the number of protons around $200 \mathrm{MeV}$ which could be produced by a laser pulse of 0.5 ps duration, $810^{20} \mathrm{~W} \cdot \mathrm{cm}^{-2}$ intensity, and $3 \mu \mathrm{m}$ FWHM focal spot irradiating a $10 \mu \mathrm{m}$ thick target. In these conditions, there would be $\sim 10^{9}$ particles in a $\Delta \mathrm{E} / \mathrm{E}=10^{-2}$ bin for a single laser shot. Since the required particle flux for treatment is in the range of $10^{10}$ particles per second [36], a laser with a repetition rate of $10 \mathrm{~Hz}$ could be appropriate. Such repetition rate is already technologically foreseeable at the needed energy levels of $\sim 100 \mathrm{~J}$ (see Figure 6.a) [37,38]. Such high laser energies could be compressed in a short pulse using available gratings in a tiled arrangement as is currently developed for future PW facilities [39].

\section{Alternative paths for proton energy increase}

There may also be alternatives to using solely solid targets as proton sources. For example, it could be ideal to have a controllable large preplasma in front of the solid. Indeed, PIC simulations have shown that a large pre-plasma increases the number and temperature of the accelerated electrons [40]. This could be achieved by using enclosures, or a flow, of controlled gas in front of the target. Another path for optimization may also be provided by the relativistically transparency regime [41] where simulations [26] show that proton acceleration should be even more efficient. In this scheme, the laser pulse interacts with the whole volume of a very thin, dense target and accelerates the whole electron population efficiently. This however requires ultra thin targets and 
therefore ultra-high temporal contrast pulses, so that the peak of the pulse interacts with an unperturbed target. We have conducted preliminary tests of such interaction using laser pulses with contrast enhanced by the use of two consecutive plasma mirrors (PM) [42]. In this configuration, we have measured $\sim 10 \mathrm{MeV}$ maximum proton energies using 30 to $100 \mathrm{~nm}$ thick targets and a peak laser

intensity of 5-9 $10^{17} \mathrm{~W} \cdot \mathrm{cm}^{-2}$ in a pulse duration of $350 \mathrm{fs}$. This is to be compared to the $1 \mathrm{MeV}$ maximum energy protons achieved for the same pulse duration and intensity in the standard regime of rear-surface acceleration, i.e. using thicker targets $(5 \mu \mathrm{m})$ [19]. However the beam quality was somewhat lower in the transparency regime than in the standard regime of rear-surface acceleration.

In this article, we attempted to give a consistent picture of the rear-surface laser acceleration regime by comparing experimental data, fluid model and PIC simulations. The study of the interaction of laser pulses with solid targets is in constant progress. New interaction regimes, such as the transparency regime, may be interesting with an increase of the maximum proton energy and a reduction of the optimal laser pulse duration, provided that a high beam quality of the proton beam is demonstrated.

\section{METHODS}

\section{Experimental configuration}

The experiments were performed using two short-pulse lasers. These were the $100 \mathrm{TW}$ laser at the Laboratoire pour l'Utilisation des Lasers Intenses (LULI), France, operating at $1.057 \mu \mathrm{m}$ wavelength, and the Atlas laser at the Max-Planck Institut für Quantenoptik, Germany, operating at $0.8 \mu \mathrm{m}$. Both 
lasers operate in the well-known chirped pulse amplification mode [43] where an initial short pulse of low energy is chirped temporally to allow amplification while minimizing spectral phase distortions and is subsequently recompressed. On both facilities, dynamic wave front correction is applied to ensure optimum and reproducible focusability at every shot. Having a constant laser focus is crucial in order to have good reproducibility of the proton beam. The focal spot full widths at half maximum (FWHM) are similar on both facilities: $6 \mu \mathrm{m}$ (LULI) and $5 \mu \mathrm{m}$ (Atlas) as measured directly by imaging diagnostics collecting light after focusing. Overall, we varied the laser energy (E) from 0.2 to $60 \mathrm{~J}$, the laser pulse duration $(\tau)$ from 150 fs to $10 \mathrm{ps}$, and the on-target intensity (I) from $10^{18}$ to $6 \times 10^{19} \mathrm{~W} \cdot \mathrm{cm}^{-2}$. The laser energy was varied by using calibrated attenuators positioned before compression, i.e. to attenuate the chirped pulse. The pulse duration was varied by changing the distance between the gratings in the compressor chamber, and was measured by second-order autocorrelation. On both facilities, the energy that precedes the main pulse is controllable within some limits by the use of fast switching Pockels cells. For this study, we kept the conditions constant and similar between the two lasers, i.e. a maximum ASE level of $\sim 1-5 \times 10^{12} \mathrm{~W} \cdot \mathrm{cm}^{-2}$, for a duration of 1.5-2 $\mathrm{ns}$ as measured by a fast photodiode. At LULI, targets are irradiated either at normal incidence or at $40^{\circ}$. At Atlas, $30^{\circ}$ incidence is used. We observed that the laser incidence on target had little influence on the resulting ion beam. This is most probably due to laser hole boring in the preformed plasma at high irradiance: regardless of the initial angle, all angles of incidence are present in the cavity bored into the dense plasma, and similar hot electron populations are thus produced. Detection of the proton beam is performed using radiochromic film (RCF) dosimetry media [44] directly exposed to the beam and Thomson parabola equipped with CR-39 [45]. RCF provides, with a high dynamic range, continuous spatial readout of the proton fluence, in coarsely resolved steps of proton energy by means of the range-energy relationship of the stopping power. Using a stack of RCF therefore allows reconstructing the spectrum of the proton beam. We observe that the 
spectra have a sharp cut-off at a maximum energy. Note that RCF is preferentially sensitive to penetrating protons, which have a large specific energy-loss and produce a high contrast image. The use of RCF to infer the proton beam spectrum has been validated by simultaneous measurements performed using a magnetic spectrometer facing the target rear side. Finally, the angularly-integrated spectrum is integrated between a fixed minimal energy, here chosen to be $4 \mathrm{MeV}$, and the maximum cut-off energy. The obtained integrated energy carried by the proton beam is then divided by the laser initial energy to obtain the laser to proton energy conversion efficiency.

\section{Numerical simulations}

In the 2D PIC simulations the peak of the linearly-polarized pulse reaches the target surface, at normal incidence, $750 \mathrm{fs}$ after the beginning of the calculation. The target is 19 microns thick, covered with a $19 \mathrm{~nm}$ layer of protons to model hydrocarbon contaminants. The initial electron temperature is $5 \mathrm{keV}$ and the proton-to-electron mass ratio is 1836. A 3 micron linear preplasma rising from $0.1 \mathrm{n}_{\mathrm{c}}$ to the target density is placed in front of the target. The target density is $2 \cdot 10^{22} \mathrm{~cm}^{-}$

${ }^{3}$. The simulation box is $134 \mu \mathrm{m}$ long and $24 \mu \mathrm{m}$ wide. The mesh size is $\Delta \mathrm{x}=\Delta \mathrm{y}=19 \mathrm{~nm}$ and there are 35 particles per species per cell, advanced with a time step of $4.2 \cdot 10^{-2}$ fs.

\section{ACKNOWLEDGEMENTS}


We acknowledge the expert support of the LULI and Atlas laser teams and useful discussion with

T. Cowan, A. Kemp and Y. Sentoku. This work was supported by DAAD, grant E1127 from Région Ile-de-France, the EU programme HPRI CT 1999-0052, and UNR grant DE-FC08-01NV14050.

COMPETING INTERESTS STATEMENT

The authors have no competing interests. 

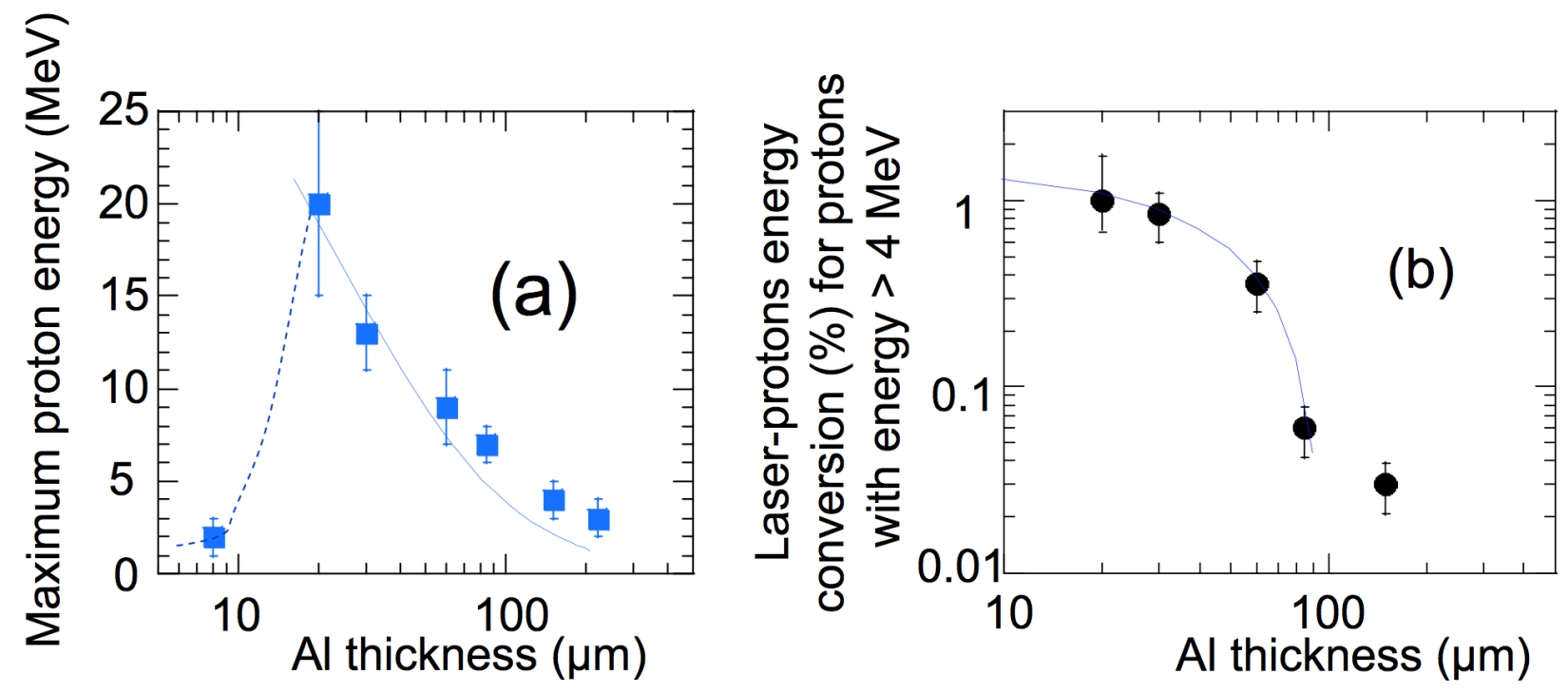

Figure 1: Thinner solid targets improve the maximum energy of laser-accelerated protons as well as the laser-to-proton conversion efficiency. (a) maximum proton energy, and (b) laser-proton energy conversion (calculated for protons with energy $>4 \mathrm{MeV}$ ) for similar laser conditions $(\tau=320 \mathrm{fs}$ and $\mathrm{I} \sim 4 \times 10^{19} \mathrm{~W} . \mathrm{cm}^{-2}$ ) and various Al foil thicknesses. Dots represent experimental data and solid lines calculations using the fluid model with the laser same laser parameters. Error bars on the proton energy represent the shot to shot fluctuation cumulated with the simulated measurement uncertainty in the detector. Error bars on the conversion efficiency take into account the maximum energy error bar, error on the laser energy, and uncertainty on the calibration of the detector that induces uncertainties on the absolute proton number. 


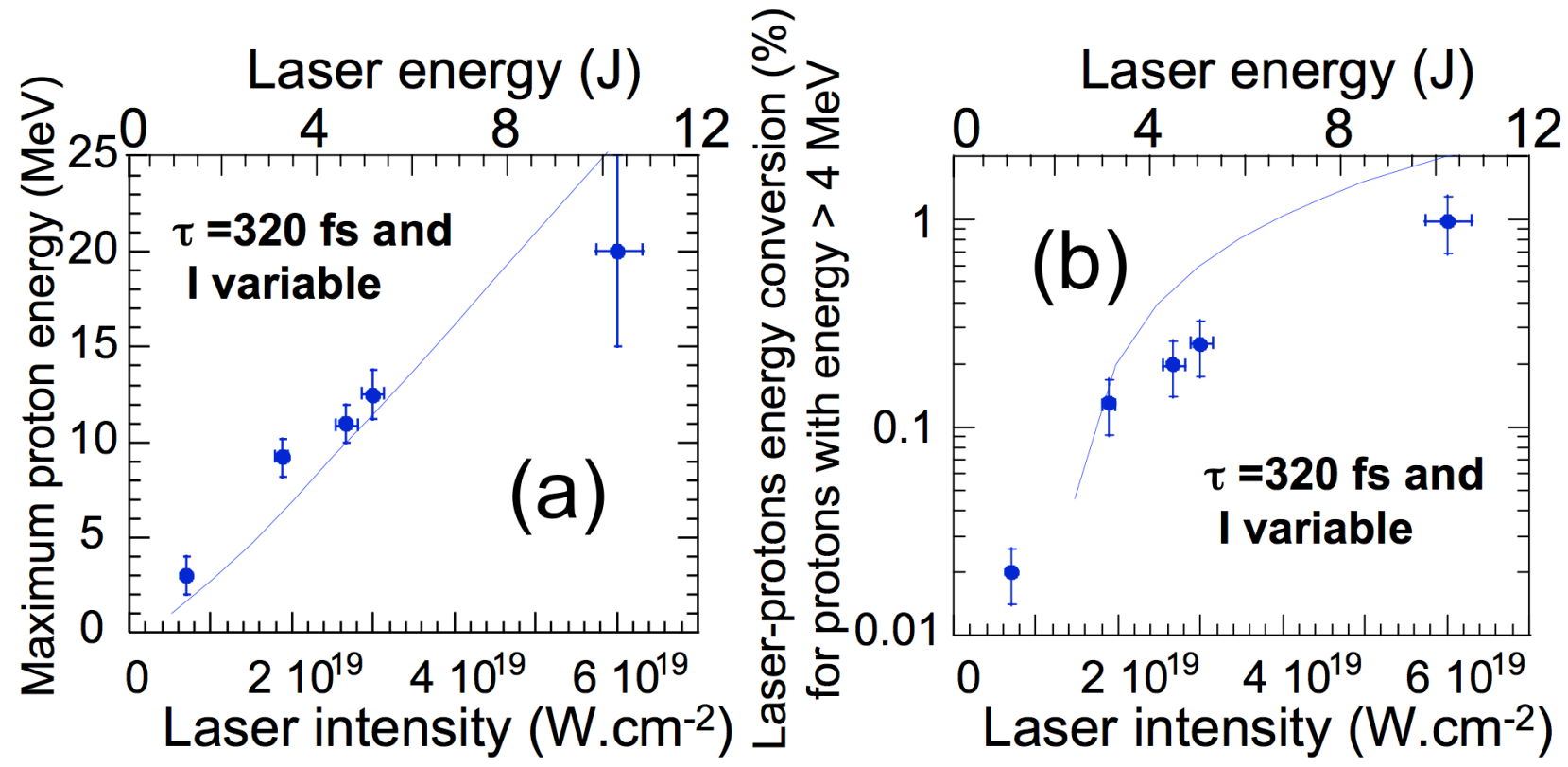

Figure 2: Laser-accelerated proton maximum energy and conversion efficiency increase with laser pulse energy. (a) maximum energy of the proton beam and (b) laser-proton energy conversion (for protons with energy $>4 \mathrm{MeV}$ ) as a function of the laser intensity (bottom) and the laser energy in the focal spot (top). The laser pulse duration is constant: $320 \mathrm{fs}$. The solid lines in (a) and (b) are fits of the data using the fluid model. Error bars on the laser energy are linked to uncertainties in the calorimeters calibration. Vertical error bars are estimated similarly as in 

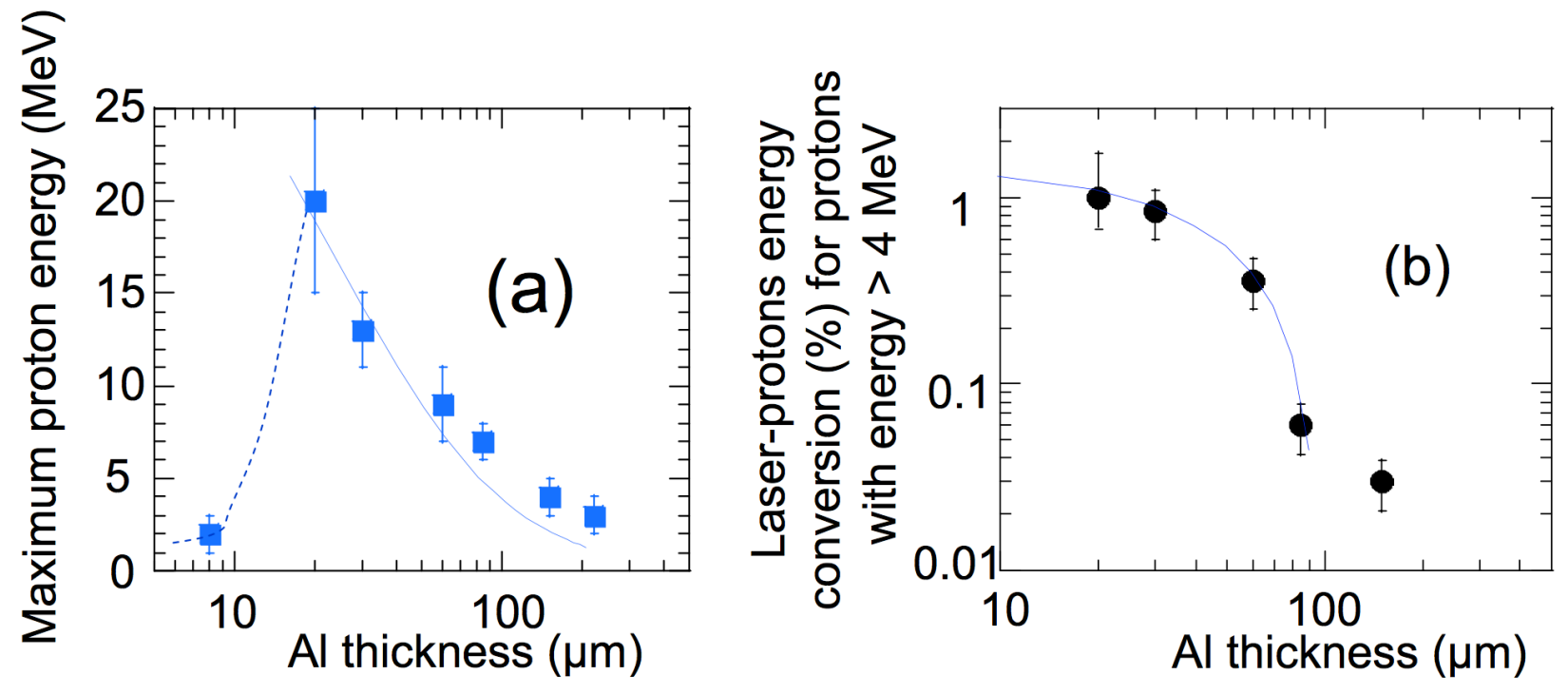

Figure 1.
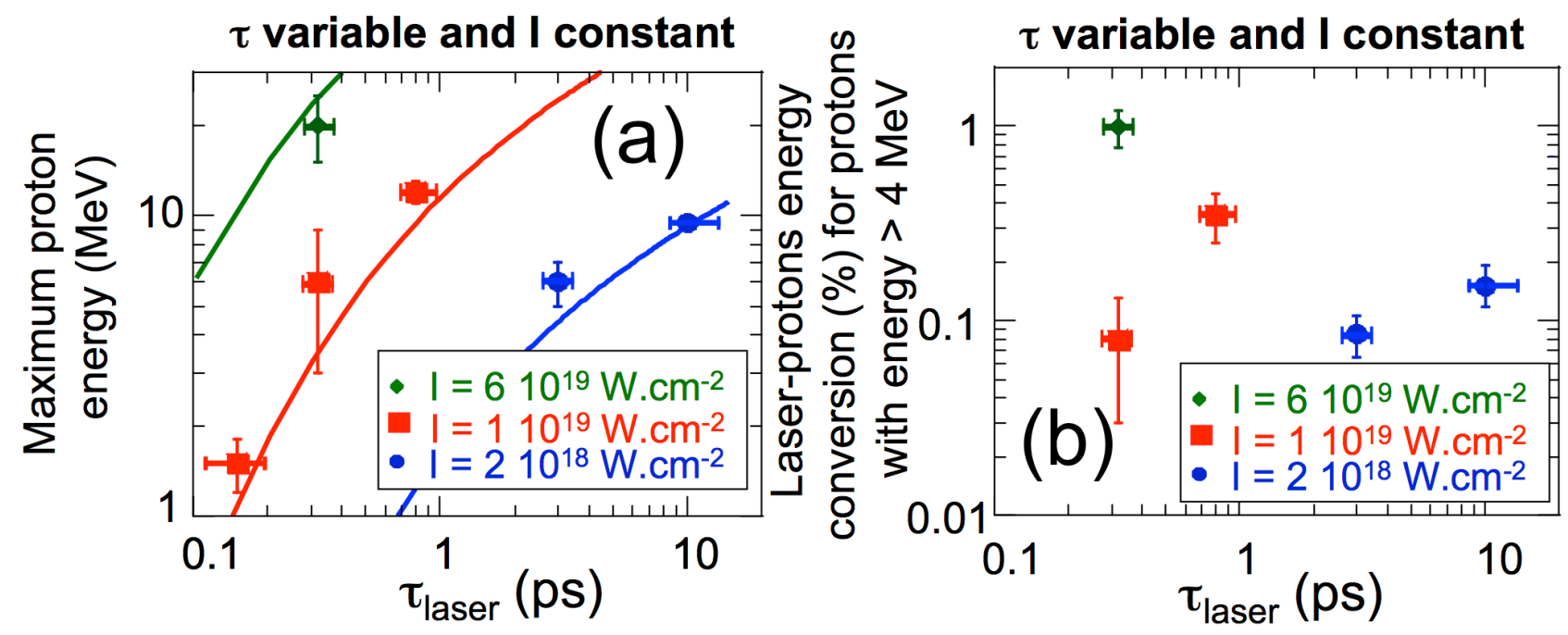

Figure 3: Longer pulses improve laser-accelerated proton maximum as well as conversion efficiency. (a) maximum energy of the proton beam and (b) laser-proton energy conversion (for protons with energy $>4 \mathrm{MeV}$ ) as a function of the laser pulse duration for three different laser intensities as indicated in the box; the laser energy is increased with the laser pulse duration to keep the laser intensity constant for each group of points. The lines are calculations for each intensity 
using the fluid model. Error bars on the laser pulse duration represent the shot to shot fluctuation combined with the estimated error linked with assuming different pulse shapes for the pulse duration retrieval. Vertical error bars are estimated similarly as in
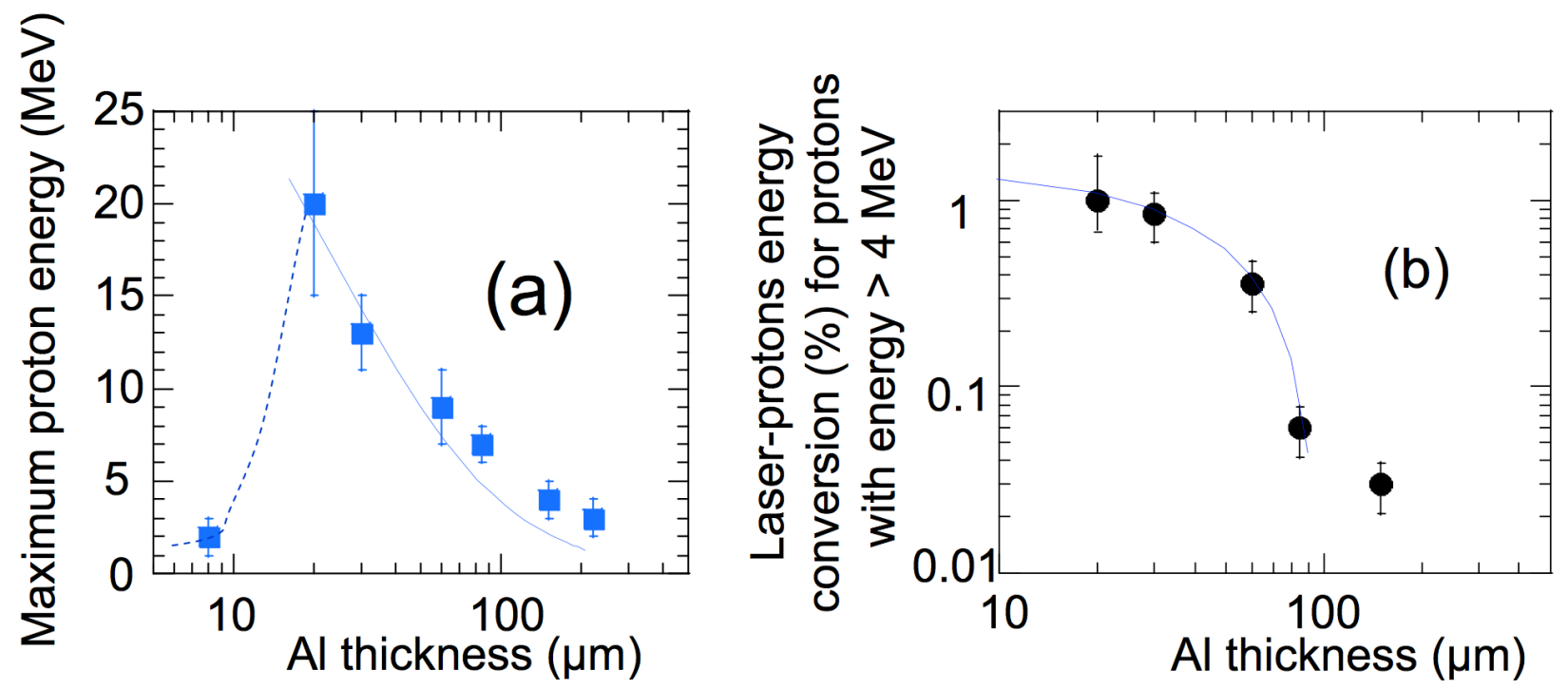

Figure 1.
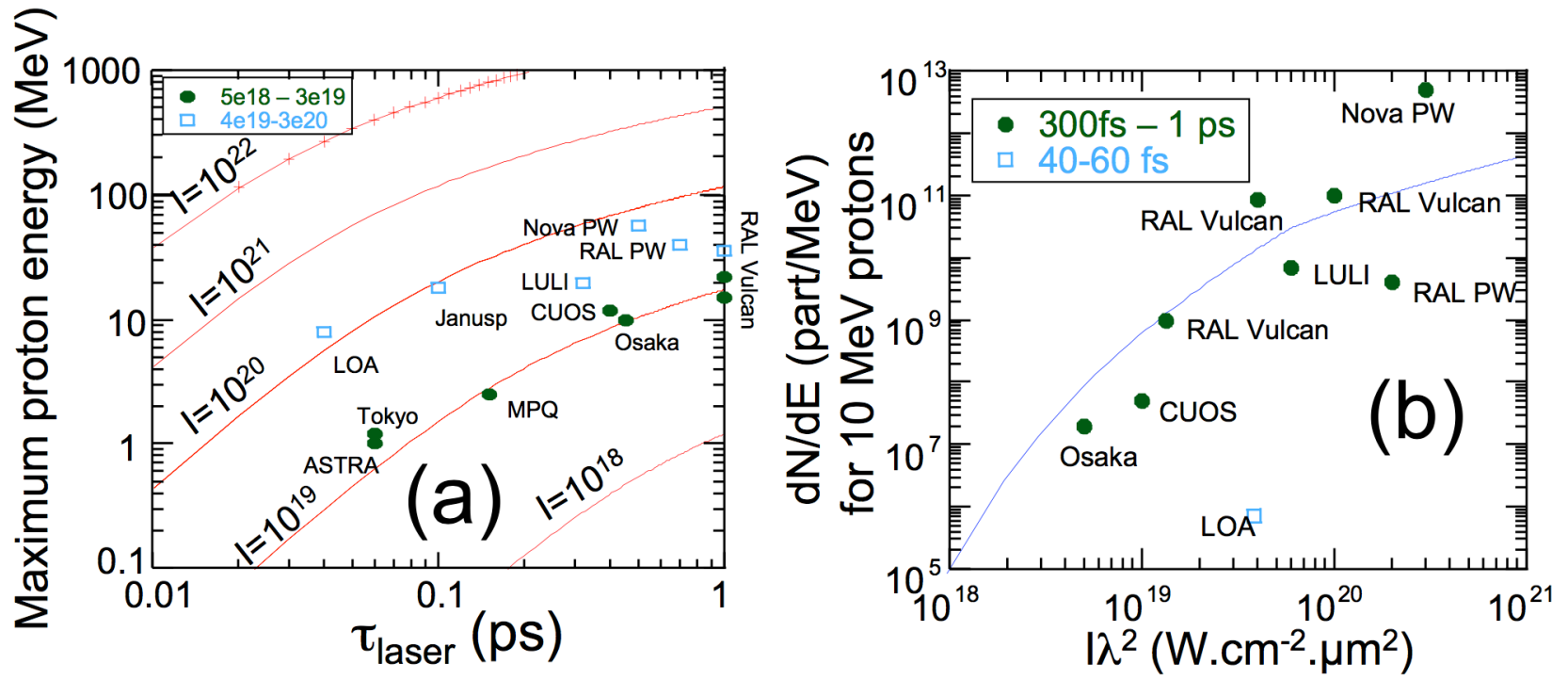

Figure 4: Comparison between fluid model predictions and previously published data. (a) maximum proton energy as a function of laser pulse duration. Dots and squares are experimental data for the two 
intensity ranges shown in the legend box, the intensities are in units of W.cm ${ }^{-2}$ ). Lines represent calculations for various laser intensities, as indicated in units of W. $\mathrm{cm}^{-2}$, using the fluid model assuming $20 \mu \mathrm{m}$ thick targets and a $10 \mu \mathrm{m}$ FWHM laser spot size. (b) Number of protons in a $1 \mathrm{MeV}$ bin around $10 \mathrm{MeV}$ as a function of laser intensity multiplied by the laser wavelength square. The last parameter is chosen since it governs the hot electron temperature $T_{p}$. Dots and squares are experimental data for the two laser pulse duration ranges shown in the legend box). The line is given by the fluid model assuming $20 \mu \mathrm{m}$ thick targets, a $10 \mu \mathrm{m}$ FWHM laser spot size and a 0.5 ps duration laser pulse. References are as follows: LOA [12], JanUSP [20], RAL PW [46], Nova PW [2], RAL VULCAN [16,17], Osaka [47], CUOS [48], MPQ [21], Tokyo [49], ASTRA [18].
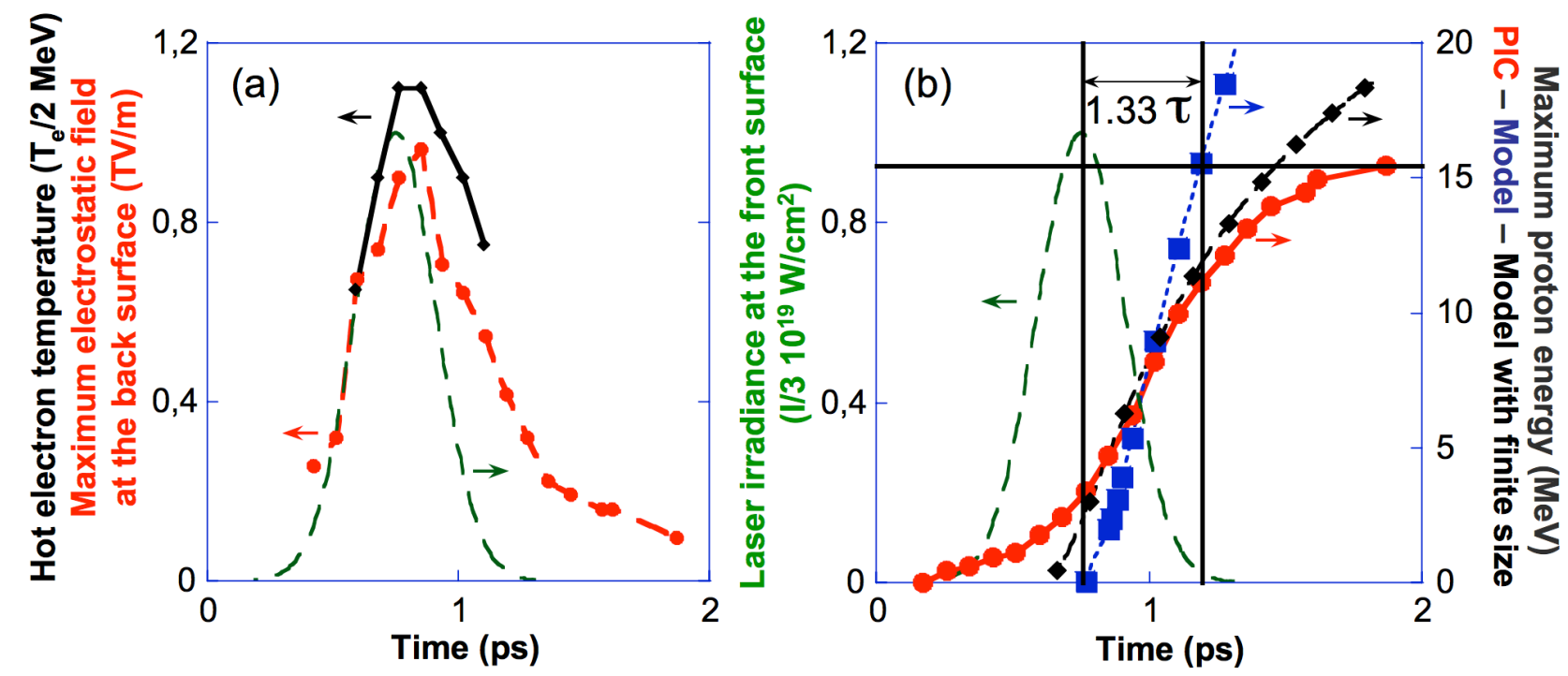

Figure 5: Study of the evolution of electron and proton population during ion acceleration using PIC and fluid simulations. (a) temporal evolution of the maximum electrostatic field at the rear of the target (red dashed line with circles) and of the hot electron temperature (black solid line with diamonds) in 2D PIC simulations. (b) temporal evolution of the maximum proton energy (red solid line with circles). The blue dotted line (with squares) corresponds to the isothermal model, that starts at the maximum of the electrostatic field, with a $0.11 \mathrm{n}_{\mathrm{c}}$ sheath density and a hot electron temperature $2.2 \mathrm{MeV}$, as given by the 
PIC simulation. The black dash-dotted line (with diamonds) corresponds to a more precise fluid calculation [50] that takes into account the finite size of the target and the energy transfer dynamics between the electrons with a finite total energy and the ions. For both plots, the dashed green line corresponds to the laser intensity at the front surface, peaking at $3 \times 10^{19} \mathrm{~W} / \mathrm{cm}^{2}$ after $750 \mathrm{fs}$. The pulse duration is $320 \mathrm{fs}$.
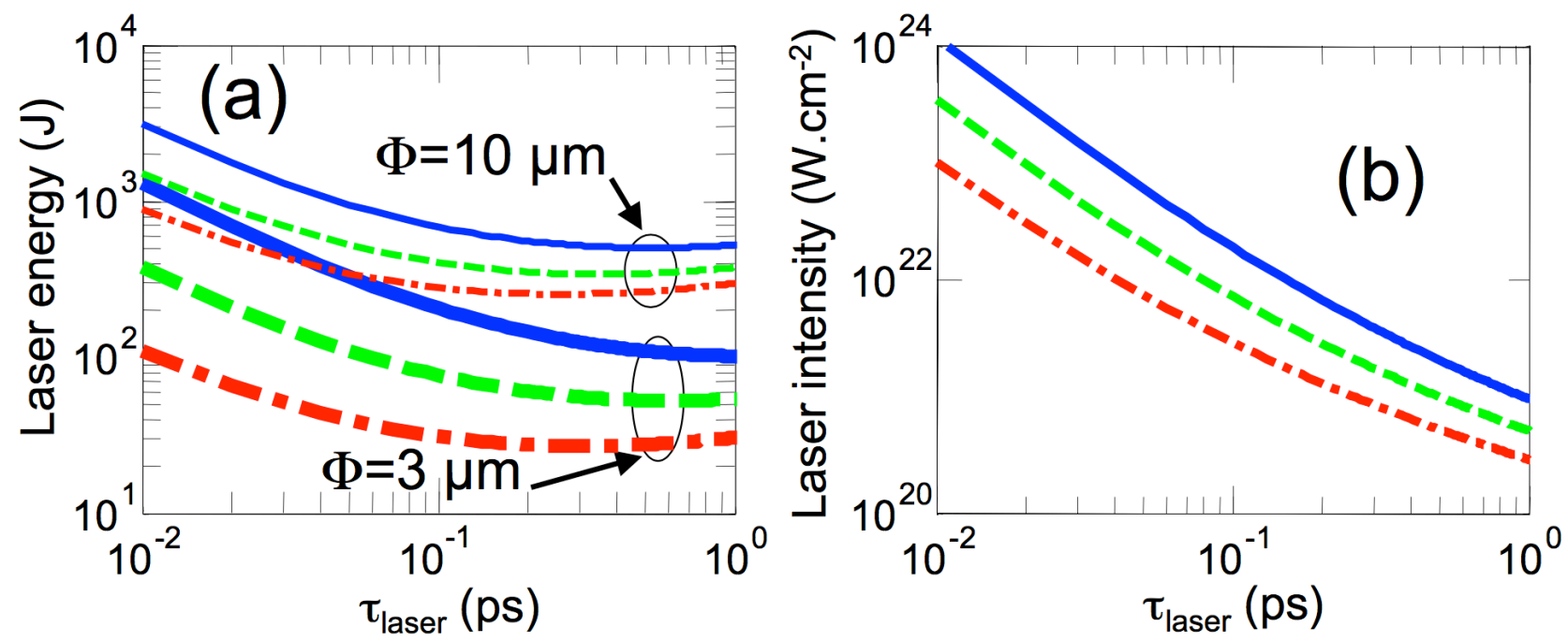

Figure 6: Projections of required laser energy and intensity to achieve a certain proton maximum energy using the adjusted fluid model. (a) required laser energy in the focal spot to achieve $200 \mathrm{MeV}$ as maximum proton energy for various laser pulse durations and target thicknesses (blue solid line: $25 \mu \mathrm{m}$, dashed green line: $10 \mu \mathrm{m}$, dotted red line: $2 \mu \mathrm{m}$ ). The thinner lines are for a $10 \mu \mathrm{m}$ focal spot, the thicker for a $3 \mu \mathrm{m}$ focal spot. (b) required laser intensity to achieve the same goal with the same parameters. Influence of the focal spot diameter is less apparent on the required intensity. 


\section{REFERENCES}

1 Clark, E., et al., Measurements of energetic proton transport through magnetized plasma from intense laser interactions with solids. Phys. Rev. Lett. 84, 670-673 (2000).

2 Snavely, R. A., et al., Intense high-energy proton beams from Petawatt-laser irradiation of solids. Phys. Rev. Lett. 85, 2945-2948 (2000).

3 Hatchett, S., et al., Electron, photon, and ion beams from the relativistic interaction of Petawatt laser pulses with solid targets. Phys. Plasmas 7, 2076-2079 (2000).

4 Gitomer, S.J., et al., Fast ions and hot electrons in the laser-plasma interaction. Phys. Fluids 29, 26792688 (1986).

5 Cowan, T., et al., Ultralow emittance, multi-MeV proton beams from a laser virtual-cathode plasma accelerator. Phys. Rev. Lett. 92, 204801 (2004).

6 Borghesi, M., et al., Multi-MeV proton source investigations in ultraintense laser-foil interactions. Phys. Rev. Lett. 92, 055003 (2004).

7 Fuchs, J., et al., Spatial uniformity of laser-accelerated ultrahigh-current $\mathrm{MeV}$ electron propagation in metals and insulators. Phys. Rev. Lett. 91, 255002 (2003).

8 Borghesi, M., et al, Electric field detection in laser-plasma interaction experiments via the proton imaging technique, Phys. Plasmas 9, 2214 (2002).

9 Patel, P., et al., Isochoric heating of solid-density matter with an ultrafast proton beam. Phys. Rev. Lett. 91, 125004 (2003).

10 Bulanov, S.V., et al., Oncological hadrontherapy with laser ion accelerators, Physics Letters A 299, 240-247 (2002).

11 Fourkal, E., et al., Particle in cell simulation of laser-accelerated proton beams for radiation therapy, Med. Phys. 29, 2788-2798 (2002). 
12 Malka, V., et al., Practicability of protontherapy using compact laser systems, Med. Phys. 31, 15871592 (2004).

13 Ledingham, K., McKenna, P., and Singhal, R., Applications for nuclear phenomena generated by ultra-intense lasers. Science 300, issue 5622, 1107-1111 (2003).

14 Ledingham, K., et al., High power laser production of short-lived isotopes for positron emission tomography, J. Phys. D: Appl. Phys. 37, 2341-2345 (2004)

15 McKenna, P., et al., Characterization of multiterawatt laser-solid interactions for proton acceleration, Rev. Sci. Inst. 73, 4176-4184 (2002).

16 Zepf, M., et al., Fast particle generation and energy transport in laser-solid interactions, Phys. Plasmas 8, 2323-2330 (2001).

17 Spencer, I., et al., Laser generation of proton beams for the production of short-lived positron emission radioisotopes, Nuc. Inst. and Methods in Phys. Research B 183, 449-458 (2001).

18 Spencer, I., et al., Experimental study of proton emission from 60-fs, 200-mJ high-repetition-rate tabletop-laser pulses interacting with solid targets, Phys. Rev. E 67, 046402-1/5 (2003).

19 Oishi, Y., et al., Dependence on laser intensity and pulse duration in proton acceleration by irradiation of ultrashort laser pulses on a Cu foil target, Phys. Plasmas 12, 073102-1/4 (2005).

20 Mackinnon, A., et al., Enhancement of Proton Acceleration by Hot-Electron Recirculation in Thin Foils Irradiated by Ultraintense Laser Pulses, Phys. Rev. Lett. 88, 215006 (2002).

21 Kaluza, M., et al., Influence of the laser prepulse on proton acceleration in thin-foil experiments. Phys. Rev. Lett. 93, 045003 (2004).

22 Hegelich, M., et al., MeV ion jets from short-pulse-laser interaction with thin foils. Phys. Rev. Lett. 89, 085002, (2002). 
23 Allen, M., et al., Direct experimental evidence of back-surface ion acceleration from laser-irradiated gold foils. Phys. Rev. Lett. 93, 265004 (2004).

24 Fuchs, J., et al., Comparison of laser ion acceleration from the front and rear surfaces of thin foils. Phys. Rev. Lett. 94, 045004 (2005).

25 Silva, L.O., et al., Proton shock acceleration in laser-plasma interactions, Phys. Rev. Lett. 92, 015002 (2004).

26 D’Humières, E., Lefebvre, E., Gremillet, L., and Malka, V., Proton acceleration mechanisms in highintensity laser interaction with thin foils, Phys Plasmas 12, 062704-1/13 (2005).

27 Esirkepov, T., Borghesi, M., Bulanov, S., Mourou, G., Tajima, T., Highly efficient relativistic-ion generation in the laser-piston regime, Phys. Rev. Lett. 92, 175003 (2004).

28 Mora, P., Plasma expansion into a vacuum. Phys. Rev. Lett. 90, 185002 (2003).

29 Gurevich, A.V., Pariiskaya, L.V., and Pitaevskii, L.P., Self-similar motion of rarefied plasma, Sov. Phys. JETP 22, 449 (1966).

30 Wilks, S. C., et al., Absorption of ultra-intense laser pulses. Phys. Rev. Lett. 69, 1383-1386 (1992).

31 Malka, G. and Miquel, J.L. Experimental confirmation of ponderomotive-force electrons produced by an ultrarelativistic laser pulse on a solid target. Phys. Rev. Lett. 77, 75-78 (1996).

32 Key, M., et al., Hot electron production and heating by hot electrons in fast ignitor research. Phys. Plasmas 5, 1966-1972 (1998).

33 Feurer, T., et al., Onset of diffuse reflectivity and fast electron flux inhibition in 528-nm-laser-solid interactions at ultrahigh intensity. Phys. Rev. E 56, 4608-4614 (1997).

34 Fourkal, E., et al., Particle selection for laser-accelerated proton therapy feasibility study, Med. Phys. 30, $1660-1670$ (2003). 
35 Esirkepov, T., et al., Proposed double-layer target for the generation of high-quality laser-accelerated ion beams, Phys. Rev. Lett. 89, 175003 (2002).

36 Khoroshkov, V. S., and Minakova, E. I., Proton beams in radiotherapy, Eur. J. Phys. 19, 523-536 (1998).

37 Hein, J., et al., Diode-pumped chirped pulse amplification to the joule level. Appl. Phys. B 79, 419422 (2004).

38 Chanteloup, J. C., et al., Overview of the Lucia laser program: towards 100 Joules, nanosecond pulses, kW averaged power, based on Ytterbium diode pumped solid State laser. Proc. SPIE 5707, 105$116(2005)$.

39 Kessler, T. J., et al., Demonstration of coherent addition of multiple gratings for high-energy chirpedpulse-amplified lasers. Opt. Lett. 29, 635-637 (2004).

40 Lefebvre, E. and Bonnaud, G. Nonlinear electron heating in ultrahigh-intensity-laser-plasma interaction. Phys. Rev. E 55, 1011-1014 (1997).

41 Fuchs, J., et al., Transmission through highly overdense plasma slabs with a subpicosecond relativistic laser pulse. Phys. Rev. Lett. 80, 2326-2329 (1998).

42 Monot, P., et al., High-order harmonic generation by nonlinear reflection of an intense high-contrast laser pulse on a plasma, Opt. Lett. 29, 893-895 (2004).

43 Strickland, D., and Mourou, G., Compression of amplified chirped optical pulses. Opt. Commun. 56, 219-221 (1985).

44 Klassen, N. V., et al., GafChromic MD-55: Investigated as a precision dosimeter. Med. Phys. 24, 1924-1934 (1997).

45 Lefevre, H.W., Sealock, R.M., and Connolly, R.C. Response of CR-39 to 2-MeV microbeams of H, He, and Ne. Rev. Sci. Inst. 53, 1221-1227 (1982). 
46 McKenna, P., et al., Characterization of proton and heavier ion acceleration in ultrahigh-intensity laser interactions with heated target foils, Phys Rev E. 70, 036405-1/6 (2004).

47 Murakami, Y., et al., Observation of proton rear emission and possible gigagauss scale magnetic fields from ultra-intense laser illuminated plastic target, Phys Plasmas 8, 4138-4143 (2001).

48 Maksimchuk, A., et al., High-energy ion generation by short laser pulses, Plasma Phys. Rep. 30, 473 495 (2004).

49 Fujii, T., et al., MeV-order proton and carbon ion acceleration by irradiation of $60 \mathrm{fs}$ TW laser pulses on thin copper tape, Appl. Phys. Lett. 83, 1524-1526 (2003).

50 Mora, P., Thin foil expansion into a vacuum, Phys. Rev. E 72, 056401-1/5 (2005) 